\title{
Counterpoint: Conventional Fluoroscopy-Guided Selective Cervical Nerve Root Block-A Safe, Effective, and Efficient Modality in the Hands of an Experienced Proceduralist
}

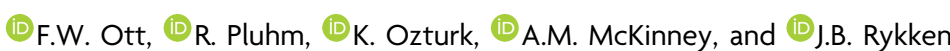

\begin{abstract}
BACKGROUND AND PURPOSE: The conventional fluoroscopy-guided (CF) selective cervical nerve root block (SCNRB) is being used commonly as a treatment for cervical radicular pain as well as a diagnostic tool. This study aimed to identify any major complications and determine the safety and clinical utility of CF-SCNRB performed in a university hospital and associated outpatient clinics.
\end{abstract}

MATERIALS AND METHODS: Two-hundred fifty-four conventional fluoroscopy-guided selective cervical nerve root blocks were retrospectively identified from 2011 to 2018 using a radiology report search tool. Each procedure was performed by an experienced neuroradiologist performing spinal injections on a full-time basis in clinical practice. A 10-point pain scale was used for pre- and postprocedural pain-level assessment. Successful conventional, fluoroscopy-guided, selective cervical nerve root block was defined as a patient-reported pain scale reduction of at least $50 \%$ and/or alleviation of numbness or paresthesia at $\geq 2$ weeks postinjection. All clinically important immediate and delayed complications were also recorded.

RESULTS: Two-hundred fifty-four conventional fluoroscopy-guided selective cervical nerve root blocks were performed via an anterolateral approach with an average fluoroscopy time of 24.3 seconds for all cases. There were no aborted procedures and no major or permanent complications. There were 14 minor complications; 12 of these were periprocedural and resolved by the 2-week follow-up visit. One-hundred eighty-five patients $(75.2 \%)$ reported pain improvement of $>50 \%$ from baseline at 15 minutes postinjection. Overall, 172 patients $(67.7 \%)$ reported $>50 \%$ pain scale reduction or alleviation from paresthesia at least 2 weeks postinjection.

CONCLUSIONS: Conventional fluoroscopy-guided selective cervical nerve root block is an efficacious, efficient, and safe outpatient procedure when performed by a skilled and experienced proceduralist.

ABBREVIATIONS: $\mathrm{CF}=$ conventional fluoroscopy; $\mathrm{CT} / \mathrm{F}=\mathrm{CT}$ fluoroscopy; SCNRB $=$ selective cervical nerve root block

$\mathbf{P}$ atients experiencing cervical and radicular arm pain, numbness, and paresthesia secondary to foraminal narrowing or impingement and resultant inflammation of the cervical nerve root may be treated with a transforaminal steroid cervical nerve root block. ${ }^{1,2}$ Selective cervical nerve root block (SCNRB) with imaging guidance, with either conventional fluoroscopy (CF) or $\mathrm{CT}$, is an intervention that has been proved effective for many patients by alleviating pain, increasing activity, improving tolerance of physical therapy, and delaying or preventing surgical intervention. $^{1,3,4}$ This procedure involves the introduction of

Received January 30, 2020; accepted after revision April 7.

From the Department of Radiology, University of Minnesota, Minneapolis, Minnesota.

Please address correspondence to Frederick W. Ott, MD, University of Minnesota Medical School, M Health Fairview, M Health Fairview Maple Grove Medical Center, 420 Delaware St SE B226, Mayo Memorial Building MMC 292, Minneapolis, MN 55455; e-mail: ottxx014@umn.edu

http://dx.doi.org/10.3174/ajnr.A6580 a needle near or into the neural foraminal perineural space, inherently carrying the risk of arterial, nerve root, and spinal cord injury. $^{1,4-7}$

Currently, CF and CT-guided SCNRBs are the most commonly available and established methods, with a trend in recent literature suggesting that CT-guided nerve blocks allow improved safety and efficacy by way of better needle tip awareness, direct visualization of the perineural space, and visualization of the vertebral and radicular arteries when using a transforaminal approach. ${ }^{2,7,8}$ However, CT-guided SCNRB may deliver a larger radiation dose to both the patient and proceduralist, ${ }^{9}$ with CT fluoroscopy (CT/F) reported to have an increase in the incurred radiation dose over CF by 19fold in the cervical region (an increase of the incremental dose of $49 \mathrm{uSv}) .{ }^{10} \mathrm{CT}$ guidance is also more costly, ${ }^{11}$ may have longer procedural times, ${ }^{2}$ and limits real-time assessment of intravascular invasion. ${ }^{3,4,8}$ Although sparse, current literature on SCNRB reports various differences in techniques via imaging medium, approach,

1112 Ott Jun 2020 www.ajnr.org 
and needle placement. While there are proponents of different techniques and modalities, most proceduralists have developed small variations that make their approach consistent and what they believe will be efficacious and safe. The authors propose that a meticulous and consistently reproducible technique and proceduralist experience are major contributors to safe and effective SCNRB.

Although relatively rare, numerous reports of complications secondary to CF and CT-guided SCNRB exist, ranging from transient arm numbness to spinal cord infarction, nerve palsies and injury, arterial injury, respiratory arrest, and death. ${ }^{1,4-8,12-14}$ While these complications have been reported in both CF- and CT-guided cases, a direct comparison of complication rates between the modalities has not been performed in a single study, to our knowledge. While injection success criteria vary from study to study, rates of patient-reported efficacy appear to be similar between CT and CF, with CT-guided efficacy rates ranging from $60 \%$ to $70.3 \%,{ }^{15-17}$ and CF-guided efficacy rates of $47 \%$ $63 \% .{ }^{3,18,19}$ Thus, the authors posit that the safety of either methodology is based on the use of a meticulous technique by an experienced proceduralist, and both imaging modalities are equally safe and effective in multiple settings on a rather consistent basis with reports of severe complications being scattered among both CT and CF-guided cases. The above warrants weighing the perceived advantages of CT against its pitfalls versus our experience of the safety and efficacy using CF. The purpose of this study was to assess the efficacy, identify any major complications, and evaluate the rate of minor complications from CF-guided SCNRB performed in a tertiary care university hospital and associated outpatient clinics.

\section{MATERIALS AND METHODS \\ Data Source}

Institutional review board approval was obtained from the University of Minnesota for this study. A retrospective analysis using the radiology information system/PACS, the electronic medical record, and the Vitrea Intelligence search tool (Vital Images, a subsidiary of Canon Group) allowed searching selected imaging reports using desired keywords. All procedures performed were in accordance with the ethical standards of the institutional research committee and with the 2013 revised Helsinki declaration and its later amendments or comparable ethical standards.

\section{Inclusion and Exclusion Criteria}

After searching the data base using the radiology information system/PACS search tool for cervical nerve root injection cases, all patients who received a CF-SCNRB from 2011 to 2018 were collected. All patients with a procedural report and postprocedural follow-up clinic visit of $>2$ weeks postinjection were included. Each procedure was performed by a single experienced neuroradiologist actively performing spinal injections on a full-time basis in clinical practice. Patients with injections lacking a comparative pre- and postprocedural patient-reported pain scale or postprocedural clinical follow-up at $>2$ weeks after SCNRB indicating nerve root block efficacy were excluded. For this study, a successful SCNRB was defined as a patient-reported pain scale reduction of at least $50 \%$ and/or alleviation of numbness or paresthesia at $\geq 2$

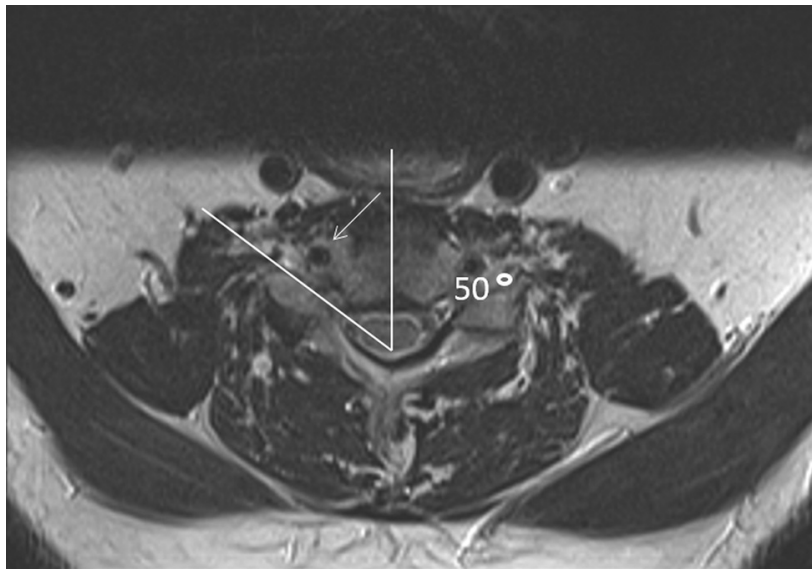

FIG 1. The foraminal angle is $50^{\circ}$. White arrow demonstrates the vertebral artery.

weeks postinjection. The definition of pain reduction was selected as an objective metric to reflect a cutoff for what is thought to constitute adequate pain relief, and this pain reduction metric has been used previously. ${ }^{16}$

\section{Author Technique}

There are many ways to perform SCNRB. The technique described herein is naturally based on the premise of safety first, but also with a requirement of efficiency, given our busy spine injection service with procedural time slots scheduled for 30 minutes. As a word of caution, SCNRBs should not be performed until the proceduralist has performed a large number of lumbar transforaminal epidural/nerve root injections and is confident and comfortable steering a spinal needle into a 1- to 2-mm space. At the participating institution, all patients undergoing SCNRB must have undergone either cervical spine MR imaging, a CT angiogram of the neck, or contrast-enhanced CT of the neck within 2 years before the procedure so that the location of the vertebral artery within the neural foramen can be identified. The vertebral artery should be located in an anterior position in the neural foramen so that the spinal needle can be safely placed posteroinferiorly. The exact angle of the neural foramen is then measured at the desired level (Fig 1) on the available cross-sectional study. Verbal and written consent is obtained from the patient, including statements of the risks of bleeding, infection, nerve root injury, vertebral or carotid artery injury, cord infarct, and stroke. The patient is then positioned at approximately a $45^{\circ}$ angle with a wedge-shaped sponge behind the back on the fluoroscopy table with the desired side of the neck for the planned injection facing upward (Fig 2A). This position requires maintaining the patient's spine in a relatively straight line so that the measured angle can be accurately reproduced at the desired level. In some cases, the patient may be positioned supine. While the supine position arguably eases the ability to maintain the spine in a straight line, it is somewhat more challenging for the proceduralist because the image intensifier will be partially in the path of access to the patient's neck, thus necessitating a table and $\mathrm{C}$-arm that can be raised in unison to get the patient positioned so the proceduralist can comfortably stand (or sit on a stool) with the patient's neck at about eye level (Fig 2B).

AJNR Am J Neuroradiol 41:1112-19 Jun 2020 www.ajnr.org 

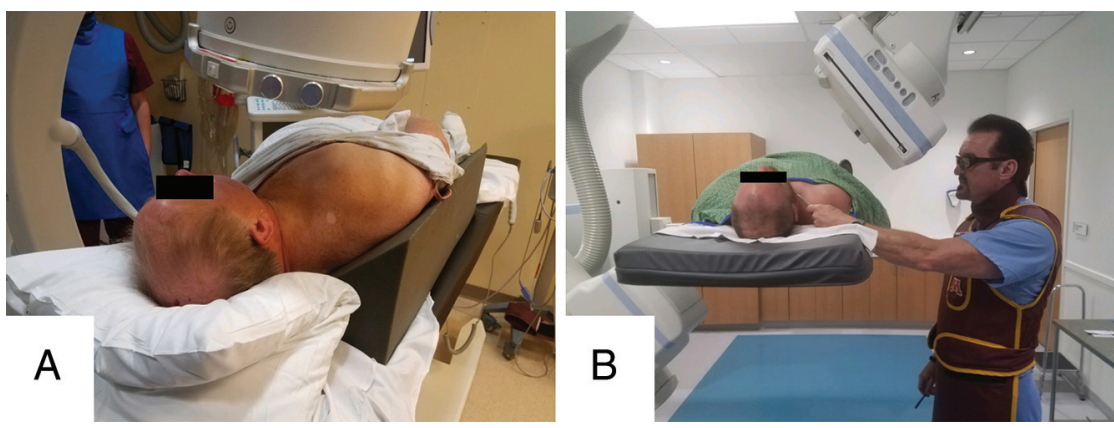

FIG 2. A, Patient positioned obliquely on a $45^{\circ}$ wedge for right-sided injection. $B$, The patient positioned supine for right-sided injection with the proceduralist using fluoroscopy to position the clamp before marking the skin. Table and C-arm are raised to eye-level to facilitate proceduralist visualization during the procedure.

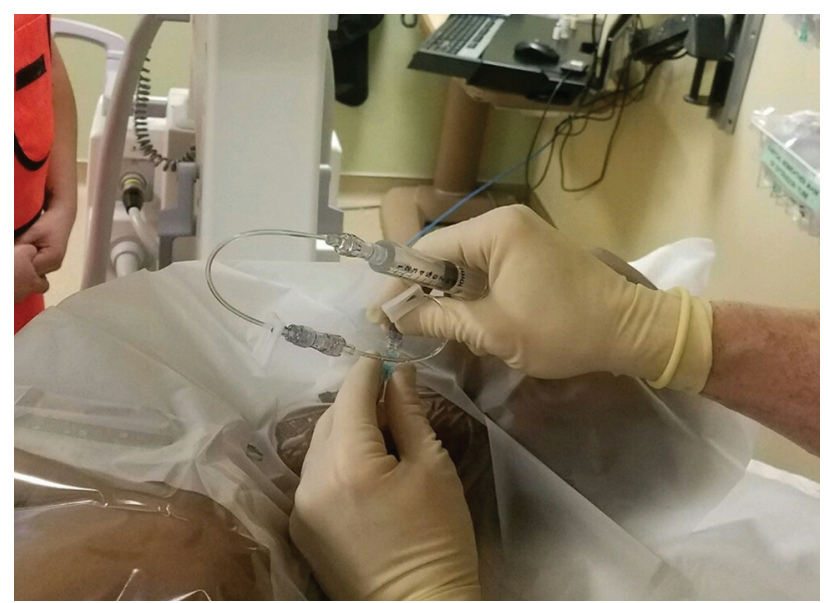

FIG 3. Contrast syringe connected via 2 short, low-volume tubing to the hub of the 25-ga spinal needle. The patient is positioned for a left-sided injection.

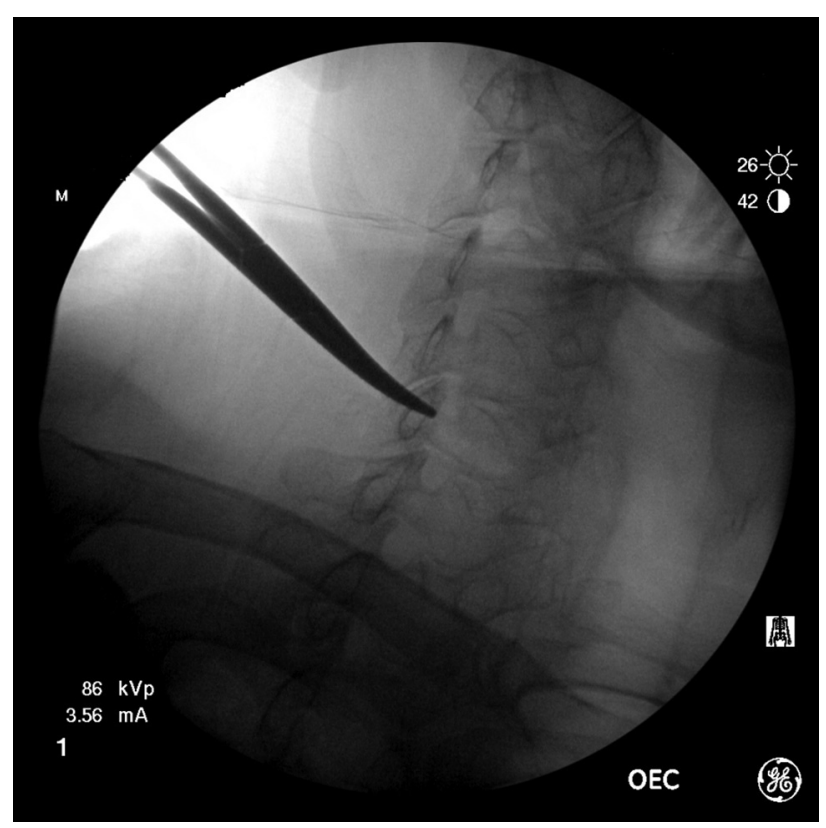

FIG 4. Curved clamp tip placed gently on the skin overlying the posterior-inferior aspect of neural foramen for optimal location, taking care to avoid indenting the skin surface.
For either approach, the straight anterior-posterior position should first be obtained with the image intensifier. If the patient is positioned on a wedge-shaped sponge, the C-arm would be positioned at approximately $45^{\circ}$ and then adjusted so that the spinous processes are centered between the lateral masses. The anterior-posterior position in the supine position should be at about $0^{\circ}$. From this position, the $\mathrm{C}$-arm is then rotated to the measured angle of the foramen. When the $45^{\circ}$ sponge position is used, the proceduralist should stand behind the patient. With the supine position, the patient should be positioned so that the affected side can be visualized with the $\mathrm{C}$-arm rotated toward the proceduralist and the range of motion adequate for the $\mathrm{C}$-arm to reproduce the desired foraminal angle from the anterior-posterior position. For either method, the patient will be positioned on the table $180^{\circ}$ in a different direction for a right- versus left-sided injection (Figs 2 and 3 , respectively).

The level of the desired neural foramen is then determined by counting down from C2. A curved Kelly clamp is then lightly positioned on the skin so that the tip is at the posterior-inferior aspect of the neural foramen (Fig 4). Notably, if the clamp is pressed too firmly against the skin, then the mark will not be representative of the desired location once the skin has recoiled. The skin is then marked with a felt tip writing instrument. The neck is then sterilely prepped and draped. The skin and deeper tissues are then anesthetized with $1 \%$ lidocaine using a 5 -mL syringe and a 1.5-inch 25-ga needle. The 2 -inch needle is not used because with many patients, the tip of the needle could reach the neural foramen and potentially the vertebral artery. The syringe is aligned parallel to the x-ray beam, and the needle is advanced all the way in. After test aspiration, the needle is slowly withdrawn and $1 \%$ lidocaine is infiltrated as the needle is backed out, leaving a small wheal on the skin. After rechecking the skin mark with a sterile clamp, either a 2- or a 3.5-inch 25-ga spinal needle with the stylet in place is advanced, while checking the needle position along the way with fluoroscopy (Fig 5). The 2-inch needle is preferred for improved control during the approach, but patients with a thicker neck require the 3.5 -inch needle. The neural foramina are not very deep to the skin in the cervical spine in most patients of normal body mass index, so it is prudent to check the anterior-posterior position fairly soon to judge the distance to the lateral mass.

Some proceduralists may aim at the superior articular facet, and once the bone is hit, the needle is moved anteriorly into the posterior aspect of the neural foramen. We prefer to not use this method because hitting the nonanesthetized periosteum is painful and can cause the patient to startle and move, potentially advancing the needle inadvertently. Additionally, the needle may skim along the anterior surface of the superior articular facet and enter and even pass through the neural foramen, potentially causing injury to the cord. The needle should be advanced to the edge 


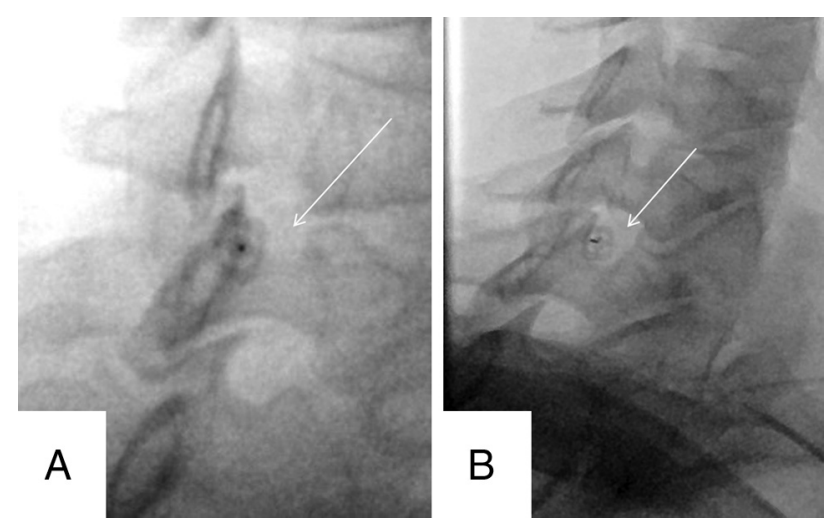

FIG 5. Ideal needle position in the posterior-inferior corner of the neural foramen ( $A$, arrow) and acceptable needle position ( $B$, arrow).

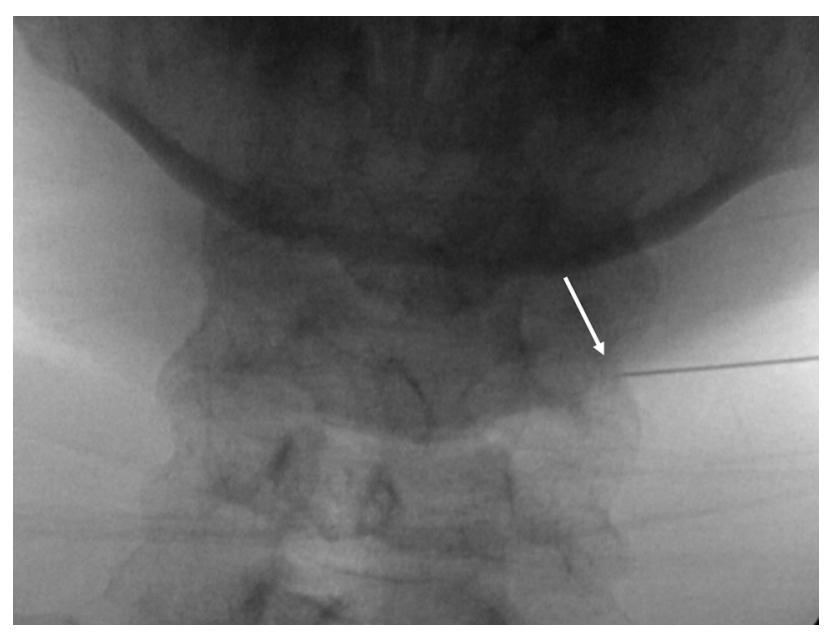

FIG 6. Needle tip advanced to the edge of the lateral mass on the anterior-posterior projection (arrow).
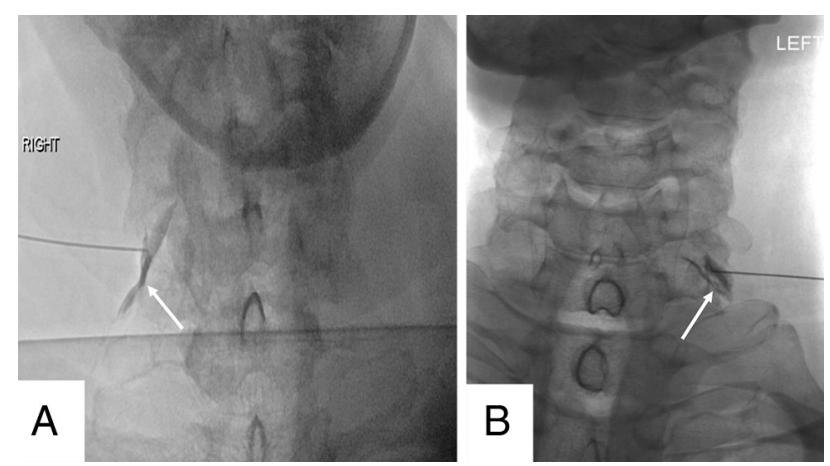

FIG 7. Contrast in the perineural space along the right $\mathrm{C} 6$ nerve root ( $A$, arrow) and clearly outlining the left $C 7$ nerve root ( $B$, arrow). Either result is acceptable.

of the lateral mass (on the anterior-posterior image) and no deeper than a few millimeters past this point (Fig 6). At this point, after removing the stylet, 2 short low-volume extension tubing (approximately $0.15 \mathrm{~mL}$ each) connected in tandem and connected to the syringe containing nonionic contrast are then attached to the hub of the 25-ga spinal needle (Fig 3). While the operator steps on the fluoroscopy pedal, a small amount of

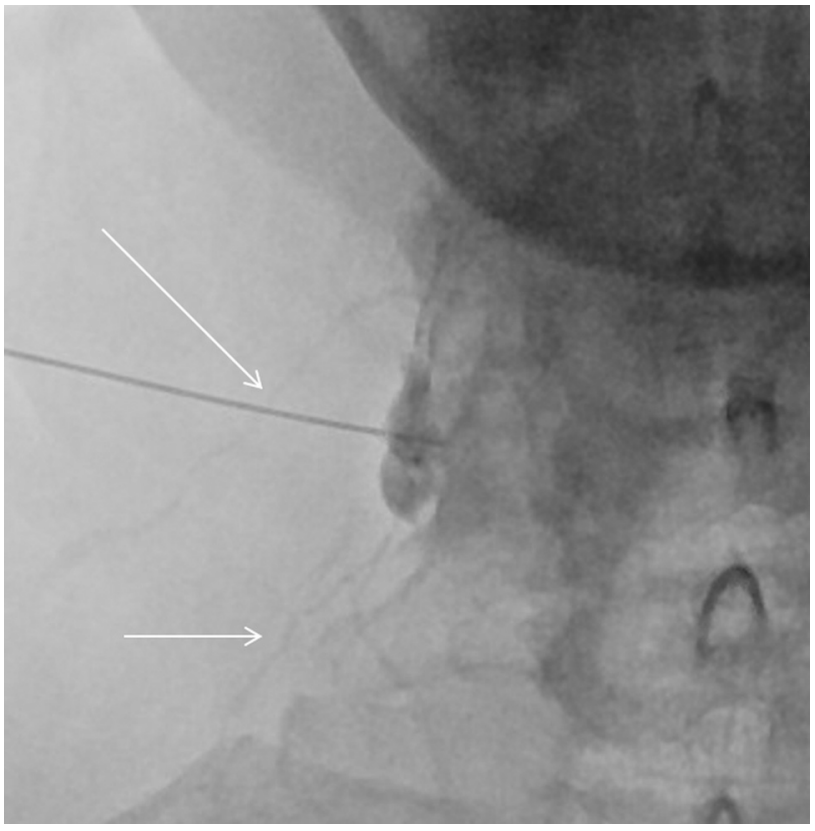

FIG 8. Vascular filling demonstrated during live injection (arrows) requiring needle repositioning.

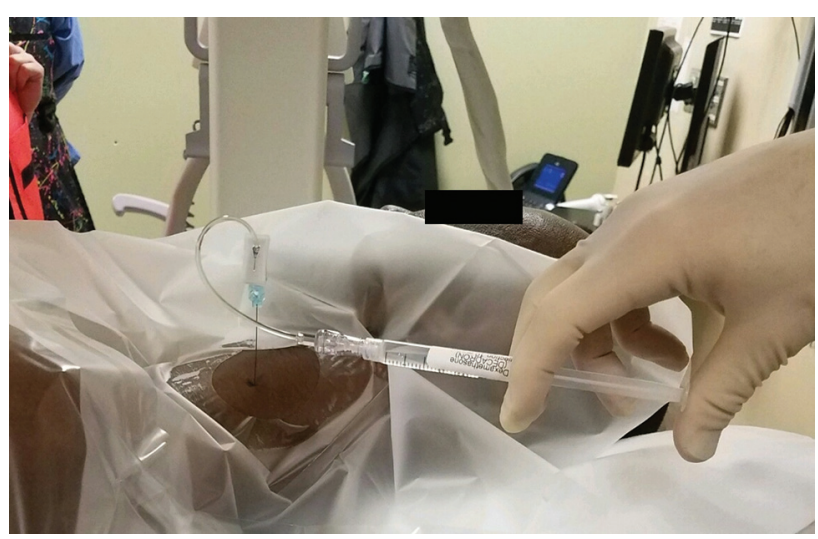

FIG 9. Syringe containing $1 \mathrm{~mL}$ of dexamethasone, $10 \mathrm{mg} / \mathrm{mL} ; 1 \mathrm{~mL}$ of $1 \%$ lidocaine; and a small amount of air connected to the remaining tubing segment closest to the hub. The syringe should be held so that the air floats anti-dependently during the injection, not horizontally as shown in this picture, which was taken before injection.

contrast is injected to outline the nerve root (Fig 7). The injection should be performed under "live" fluoroscopy to assess possible vascular filling (Fig 8). This could be missed if the contrast is injected and then the fluoroscopy activated. This is a distinct advantage of using CF over CT, permitting a large FOV while observing a live or real-time injection. This same degree of visualization is not readily achievable with CT or CT/F, particularly of import above and below the FOV with CT.

From this author's experience, advancing the needle further into the foramen only increases the chance of encountering a vessel, which requires repositioning the needle and is unnecessary to improve efficacy. Once the nerve root is outlined with contrast and there is no vascular filling, the tandem tubing is separated in the middle leaving 1 segment of tubing connected to the hub of 
Table 1: Patient demographics

\begin{tabular}{lcc}
\hline Study Population & Male & Female \\
\hline Average age $(\mathrm{yr})$ & 51.5 & 50.2 \\
No. & 123 & 131 \\
Percentage & $48.4 \%$ & $51.6 \%$ \\
\hline
\end{tabular}

Table 2: Levels of SCNRBs

\begin{tabular}{lc}
\hline Level of Injection & No. of Patients \\
\hline C4 & 9 \\
C5 & 25 \\
C6 & 115 \\
C7 & 76 \\
C8 & 29 \\
\hline
\end{tabular}

the needle (rather than disconnecting the tubing from the hub of the needle, which could potentially change the needle tip position during manipulation). A syringe containing $1 \mathrm{~mL}$ of $10 \mathrm{mg}$ / $\mathrm{mL}$ dexamethasone, $1 \mathrm{~mL}$ of $1 \%$ lidocaine, and a small amount of air is connected to the tubing segment that remains attached to the needle hub (Fig 9). After a brief test aspiration, the dexamethasone and lidocaine mixture is slowly injected. The tandem tubing is used in this fashion to minimize the chance of needle displacement when changing syringes, which could be disastrous. In addition, using 2 tubing portions connected in tandem allows an increased distance between the proceduralist's hand and the $\mathrm{x}$ ray beam during the live contrast injection. A small amount of air is kept in the syringe with the medication, held such that the air floats anti-dependently as the medication is injected. As the final amount of medication enters the tubing, it is followed by the air kept anti-dependently in the syringe until the medication gets to the hub of the needle. At this point, the injection is completed, and the needle, still connected to the tubing and syringe, is removed. With this technique, no air is injected into the patient. Even then, if it were injected, it would merely be deposited in the perineural space and eventually resorbed. Dexamethasone is used as the steroid of choice because it is a solution rather than a suspension of particles, decreasing the chance of occluding end capillaries in the brain and spinal cord. It has been shown that direct injection of dexamethasone solution into the vertebral artery of pigs produced no serious sequelae, whereas injecting particulate corticosteroids produced serious neurologic changes and infarction requiring ventilator support. ${ }^{20}$

After the procedure, the patient is assessed for any adverse reactions and then observed for 15 minutes before discharge. A 10-point pain scale is used for pre- and postprocedural pain-level assessment. Notably, these procedures are performed without the patient under conscious sedation. This helps minimize the patient's time at the facility, avoids the risk of sedation, permits quick feedback during the procedure, and allows more rapid identification of any complications.

\section{RESULTS}

All 254 injections were technically successful and without permanent adverse sequelae. Of the 254 cases, 131 patients were women and 123 men (Table 1). The average ages of men and women included in the study were 51.5 and 50.2 years, respectively (Table 1).
There were 9 injections at $\mathrm{C} 4$, twenty-five at $\mathrm{C} 5$, one hundred fifteen at C6, seventy-six at C7, and 29 at C8 (Table 2). The average fluoroscopy time for all cases was 24.3 seconds. Three patients reported a brief vasovagal reaction following the injection, all of whom achieved resolution of symptoms within a few minutes postinjection with conservative measures such as flat positioning and oral fluids. Another patient (right C6 nerve root blockade) had transient pseudo-Horner syndrome with self-reported right-sided blurry vision as well as right-sided ptosis and a constricted pupil. This patient's symptoms resolved within 30 minutes of injection, and with clinical evaluation, she was diagnosed with anesthetic-related sympathetic blockade due to inadvertent epidural extension of the injection with resultant anesthetization of the $\mathrm{C} 8-\mathrm{T} 2$ nerve roots as they traversed the epidural space on their way to the neural foramina. Two more patients reported diffuse headaches days after the procedure that each attributed to the cervical nerve root blockade. Both patients reported resolution at their next follow-up.

There were no aborted procedures and no major or permanent complications, which would include cerebral, cerebellar, brain stem, or spinal cord infarction, permanent nerve injury, and death. Of note, transient arm numbness was not included as an untoward effect because it is expected when exposing cervical nerve roots to a lidocaine-containing mixture.

There were 14 events reflecting minor complications, potential complications, or adverse effects (Table 3). Twelve (4.7\%) patients had transient minor adverse reactions to the injection, while $2(0.8 \%)$ reported delayed minor adverse reactions, which also resolved by 2 weeks at follow-up. Of the 254 patients questioned at the 15-minute interval, 8 reported a mild increase from their usual pain in a familiar distribution. Of these, all 8 had resolution of that pain at the 2-week follow-up.

One hundred eighty-five patients $(75.2 \%)$ reported pain improvement of $>50 \%$ from baseline at 15 minutes postinjection, while 61 patients $(24.8 \%)$ reported a reduction of $<50 \%$ from baseline pain at 15 minutes. For the improved-pain group, the average pain score preinjection was 4.7/10 before injection and 0.7/ 10 at 15 minutes postinjection, while the nonimproved group averaged a preinjection pain score of $5.2 / 10$ and a postinjection score of $4.4 / 10$.

Of the 185 patients with $>50 \%$ pain reduction reported at 15 minutes postinjection, 139 (75.1\%) reported maintenance of meaningful pain reduction below their baseline at the 2-week follow-up. Forty-six (24.9\%) of the 185 patients with an adequate response at 15 minutes postinjection reported that their pain had returned to baseline at 2 weeks.

Of the 61 patients with $<50 \%$ pain reduction reported at 15 minutes postinjection, $26(42.6 \%)$ reported meaningful pain reduction below their baseline at the 2 -week follow-up. Thirtyfive $(57.4 \%)$ of the 61 patients with an inadequate response at 15 minutes postinjection reported that their pain had remained unimproved at 2 weeks. Seven of the 8 patients (87.5\%) with radiculopathy without pain also reported resolution of their symptoms 2 weeks postinjection (Table 3 and Fig 10). Overall, 172 of the 254 patients $(67.7 \%)$ reported $>50 \%$ pain scale reduction or alleviation from paresthesia at least 2 weeks postinjection. 
All fluoroscopy times for CF-

\begin{tabular}{lr}
\hline Primary outcomes & \\
$>50 \%$ pain relief at $15 \mathrm{~min}$ & $185(75.2 \%)$ \\
$<50 \%$ pain relief at $15 \mathrm{~min}$ & $61(24.8 \%)$ \\
$>50 \%$ pain reduction from baseline at $2 \mathrm{wk}$ & $165(67.1 \%)$ \\
$<50 \%$ pain reduction from baseline at $2 \mathrm{wk}$ & $81(32.9 \%)$ \\
No preprocedural pain (patient-reported numbness or paresthesia) & $8(3.1 \%)$ \\
Alleviation of preprocedural numbness or paresthesia at $2 \mathrm{wk}$ & 7 of $8(87.5 \%)$ \\
Minor adverse reactions or events & $3(1.2 \%)$ \\
Vasovagal reaction & $1(0.4 \%)$ \\
Transient pseudo-Horner syndrome (sympathetic blockade) & $2(0.8 \%)$ \\
New onset of diffuse headache & $8(3.1 \%)$ \\
Increase in usual pain in a familiar distribution & \\
\hline a Transient arm numbness postinjection was not included as an untoward effect because it is expected when \\
exposing cervical nerve roots to a lidocaine containing mixture.
\end{tabular}

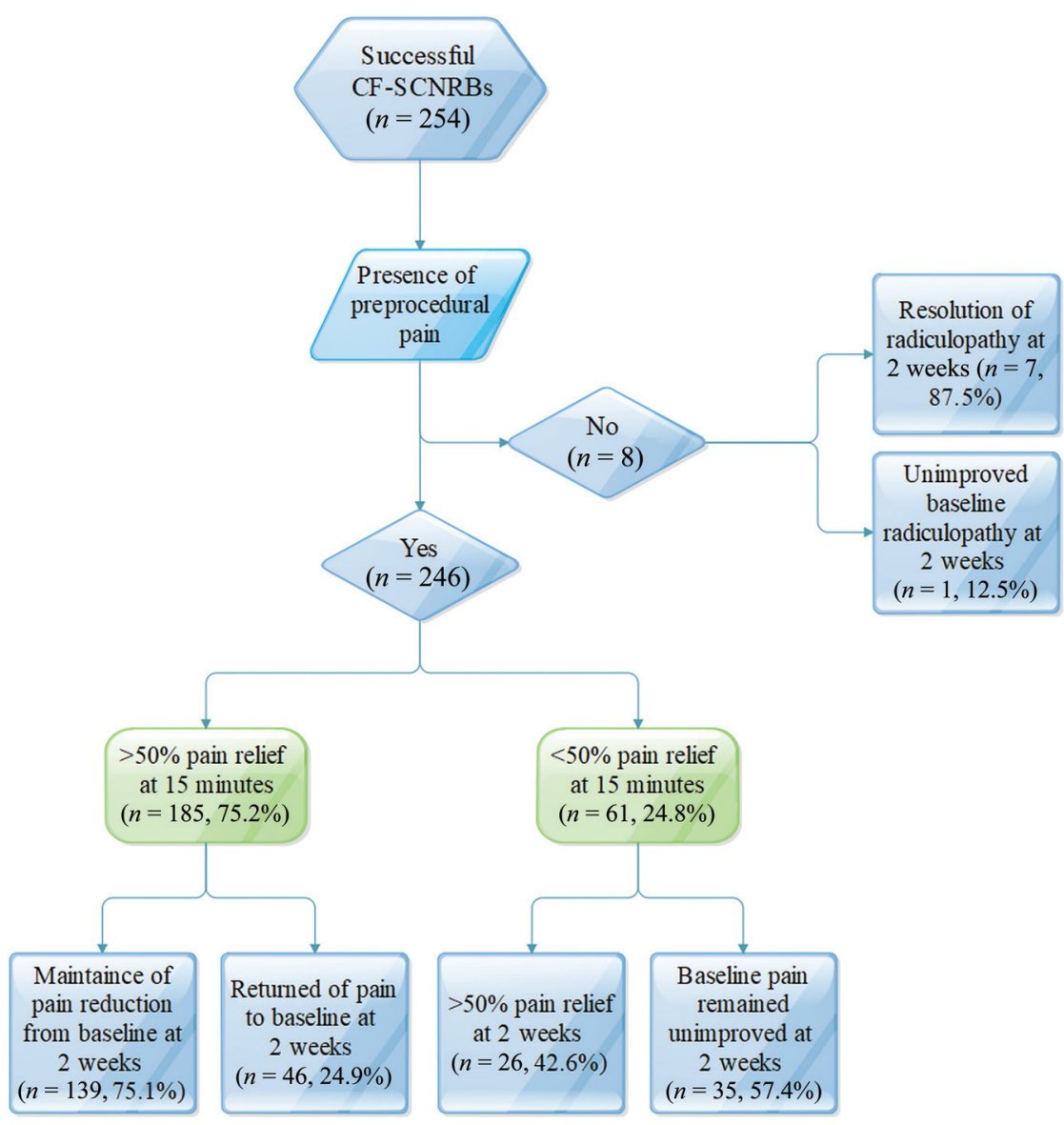

FIG 10. Flow chart demonstrates the effectiveness of CF-SCNRB with regard to postprocedural pain assessment at 15 minutes and 2 weeks.

Table 4: Radiation exposure based on time ${ }^{a}$

\section{Approximate Corresponding Radiation} Incurred

CT-guided average effective dose CF average fluoroscopy time of $24.3 \mathrm{sec}$

$0.36 \pm 0.41 \mathrm{mSv}$ $0.17 \mathrm{mSv}$

${ }^{a}$ Average CT-guided effective dose was calculated from dose-length product values reported by Lazarus et al ${ }^{11}$ over 228 SCNRB cases using a conversion factor of $5.4 \mathrm{uSv} / \mathrm{mGy} \times \mathrm{cm}$ for cervical spine examinations developed by Huda et al. ${ }^{21} \mathrm{CF}$ radiation dose is referenced and extrapolated from a direct comparison by Schmid et $\mathrm{al}^{27}$ using an Alderson Rando phantom.
No. of Patients

SCNRB were recorded at under 2 minutes, with a range of $7-78 \mathrm{sec}$ onds. The fluoroscopy time averaged 24.3 seconds per procedure, and radiation exposure based on the time is shown in Table 4 and compared with an average effective dose for CT-guided interventions in a study by

Lazarus et $\mathrm{al}^{11}$ using a conversion factor developed by Huda et al. ${ }^{21}$

\section{DISCUSSION}

This investigation supports the supposition that CF-SCNRBs, when performed by an experienced provider and with meticulous technique, are equivalent in safety and efficacy compared with existing CT-SCNRB publications. First, no major complications occurred, and there was a minor complication rate $(5.5 \%)$ comparable with the CF rate $(5.3 \%)$ of Pobiel et $\mathrm{al}^{3}$ and with a CT-guided series (4\%). ${ }^{22}$ Slight variations of postinjection minor complication rates between our study and existing publications could be explained by different definitions of what is considered an expected or adverse reaction (eg, includingversus-excluding increased radicular pain). ${ }^{23}$ Our expected symptoms including mild injection site pain, lightheadedness on standing, and transient numbness in an expected distribution, are similar to those in other publications with minor adverse event rates. ${ }^{3,15,22,23}$ Hence, the authors of this study opine that the lack of major complications and comparative minor adverse event rates in this study are products of meticulous adherence to a proved technique performed by an experienced neuroradiologist.

The use of a nonparticulate steroid likely also plays a role in the safety of our technique. A review of complications during cervical nerve root injections by Scanlon et $\mathrm{al}^{5}$ supports an embolic mechanism possibly being the most common cause of major complications, with an inadvertent intra-arterial injection of particulate steroid causing a distal infarct. The ability to fully assess real-time intravascular invasion paired with the use of a nonparticulate steroid, in our experience, renders CF guidance along with a dexamethasone-based injection mixture the safest and most efficient option. Bartleson and $\mathrm{Maus}^{24}$ reported that with the acquisition of a 
preprocedural imaging study such as MR imaging or CT angiography, appropriate anatomic understanding of each patient could be achieved, especially the location of the vertebral artery in the neural foramen. Pobiel et $\mathrm{al}^{3}$ also discussed how real-time visualization of the contrast injection with $\mathrm{CF}$ is more advantageous in preventing accidental arterial injection than increased anatomic visualization during needle tip guidance via CT. This is also recommended by others including Palmer ${ }^{25}$ and accentuated by the presentation by Hodler et $\mathrm{al}^{4}$ of 2 cases with the disastrous complication of tetraplegia secondary to ischemic myelopathy during procedures performed under CT guidance. We do not use DSA to assess vascular filling, and it has been recently reported that the detection rate of intravascular injection during real-time fluoroscopy shows no statistical difference compared with the detection rate during DSA when performing SCNRB. ${ }^{26}$

This study demonstrated that CF-SCNRB could be as effective as CT-SCNRB if performed correctly. We believe our criteria for adequate pain reduction were stringent, with a minimum of $50 \%$ patient-reported decrease in usual pain or continued alleviation of radiculopathy and paresthesia at a follow-up clinic visit after at least 2 weeks to be considered successful. This metric has been used as a fair indication of significant pain relief previously, ${ }^{16}$ potentially aiding rehabilitation and hopefully avoiding the need for surgical intervention. At $\geq 2$ weeks, 172 of 254 patients $(67.7 \%)$ reported a $>50 \%$ reduction in usual pain or complete alleviation of paresthesia in this study. In comparison with the success rates found in the existing CT-SCNRB series, our efficacy rate was favorable. ${ }^{15-17}$

A study in 2014 found that CT/F-guided SCNRB increased the incurred radiation dose up to 19 -fold in the cervical region and 8.0-fold in the lumbar region (incremental doses of 49 and $140 \mathrm{mSv}$, respectively) relative to CF. ${ }^{10}$ Another disadvantage of CT guidance is added cost to the patient. ${ }^{11,25} \mathrm{CT}$ guidance has been reported as approximately 0.9 relative value units higher than CF (3.32 for CT for therapy guidance versus 2.41 for CFguided spinal injection). ${ }^{11} \mathrm{~W}$ ith safety and efficacy equivalence, this study suggests that CF is the more practical technique by way of potentially reduced procedural times and radiation exposure for both the patient and the proceduralist. ${ }^{11,21,25,27}$

Nevertheless, there is a recent publication by Dietrich et $\mathrm{al}^{28}$ that suggests that radiation exposure for the proceduralist may be higher with $\mathrm{CF}$ than with CT/F-guided lumbar procedures. However, the authors indicated that during most CT/F acquisitions, the proceduralist is positioned behind the side of the gantry, while during most acquisitions with CF guidance, the operator is directly adjacent to the patient. The authors state that "fluroscopyguided lumbar spine injections necessitate real-time manual guidance and manipulation of the needles; thus, the body, wrist, and hand of the interventionalist is[sic] exposed to scattered radiation due to the proximity of the primary x-ray beam." ${ }^{28}$ That seems to indicate that the proceduralist was actively using fluoroscopy while guiding the needle and a "plastic forceps" was used to keep his or her hand out of the beam. With our technique, we do not use live fluoroscopy while steering the needle; rather, we triangulate, advance the needle, and then check the needle position by tapping the fluroscopy pedal. As a consequence, we are uncertain as to whether their study design applies to our technique. The only real- time exposure in our technique would be during contrast injection, which we strongly recommend. This study also reports a higher dose to the patient during CT versus CF guidance for lumbar transforaminal injections, at $0.33 \pm 0.1$ and $0.24 \pm 0.22 \mathrm{mSv}$, respectively. Although these data are from lumbar injections and cervical injections may yield higher differential exposures, this is a significantly smaller difference than had been previously stated, which is likely due to use of newer low-dose CT applications.

Although a procedural time advantage with CF has been reported, to our knowledge, there is no study that directly compares CT-guided procedural times with $\mathrm{CF}$ procedural times. With the wide availability of CT/F, especially for the highly skilled proceduralists frequently performing these injections, and presuming conservative use of fluoroscopy, a significant procedural time advantage using CF guidance seems unlikely, though this may be worthy of further investigation.

There are certain limitations to our study design. The retrospective nature of this study relies heavily on the accuracy of consultation and procedural notes, as well as appropriate patient reporting of events surrounding and at the time of injection. Although we are confident that a major complication as a result of an injection would be made known to our department, it is conceivable that additional minor complications occurred that were not properly reported by patients or recorded in consultation or procedural notes. Notably, the injection levels in this patient population are generally in the mid and lower cervical spine. Thus, applying the same generalization regarding safety to upper cervical nerve root injections should be made with caution. Also, the efficacy of our injections did not account for a vertebral level or classification of the severity of foraminal stenosis, which may be a useful area of study in the future. Last, because the injections in this study were performed by a single proceduralist, further evaluation of safety using data from injections performed by multiple proceduralists using the same technique would be of interest.

The efficacy rate in this study is similar to that of CT-guided SCNRB. This finding combined with the small percentage of minor adverse events and the efficiency of our methodology render CF a viable option for SCNRB. Recent reports in the literature suggest that $\mathrm{CT}$ guidance provides better visualization of anatomic landmarks relative to the needle tip, which arguably reduces the risk of major complications; however, catastrophic neurovascular complications during CT-guided SCNRB injections have occurred. In any event, the reports of major neurovascular complications for either CF or CT guidance are extremely rare and a sufficiently powered prospective study comparing major complication rates between modalities would likely not be possible. To date, using this technique, which has been routinely repeated by 2 additional neuroradiologists in our group, we have yet to encounter a major complication in our own practice.

\section{CONCLUSIONS}

Whether using CF or CT guidance for cervical nerve root injections, there is a low incidence of minor complications $(4 \%-6 \%)$ and serious complications are exceedingly rare, given the large number of these procedures performed annually. This study confirms that CF is as safe and effective as CT for the guidance of SCNRB when 
using a meticulous technique and a nonparticulate steroid and performed by an experienced and skilled proceduralist. The lack of meaningful superiority in these areas by existing CT-guided datasets in conjunction with the increased patient radiation exposure and cost to the patient inherent in CT makes CF the favorable technique in our practice.

\section{REFERENCES}

1. Rozin L, Rozin R, Koehler SA, et al. Death during transforaminal epidural steroid nerve root block (C7) due to perforation of the left vertebral artery. Am J Forensic Med Pathol 2003;24:351-55 CrossRef Medline

2. Wolter T, Mohadjer M, Berlis A, et al. Cervical CT-guided, selective nerve root blocks: improved safety by a dorsal approach. AJNR Am J Neuroradiol 2009;30:336-37 CrossRef Medline

3. Pobiel RS, Schellhas KP, Eklund JA, et al. Selective cervical nerve root blockade: prospective study of immediate and longer term complications. AJNR Am J Neuroradiol 2009;30:507-11 CrossRef Medline

4. Hodler J, Boos N, Schubert M. Must we discontinue selective cervical nerve root blocks? Report of two cases and review of the literature. Eur Spine J 2013;22(Suppl 3):466-70 CrossRef Medline

5. Scanlon GC, Moeller-Bertram T, Romanowsky SM, et al. Cervical transforaminal epidural steroid injections: more dangerous than we think? Spine (Phila Pa 1976) 2007;32:1249-56 CrossRef Medline

6. Brouwers PJ, Kottink EJ, Simon MA, et al. A cervical anterior spinal artery syndrome after diagnostic blockade of the right C6-nerve root. Pain 2001;91:397-99 CrossRef Medline

7. Chang MC. Spinal cord injury by direct damage during CT-guided C7 transforaminal epidural steroid injection. Am J Phys Med Rehabil 2018;97:e62-64 CrossRef Medline

8. Keir A, Pandey UC, Cheri T, et al. Respiratory arrest following CT guided selective cervical nerve root injection. Crit Care Shock 2018;21:99-102

9. Nawfel RD, Judy PF, Silverman SG, et al. Patient and personnel exposure during CT fluoroscopy-guided interventional procedures. Radiology 2000;216:180-84 CrossRef Medline

10. Maus T, Schueler BA, Leng S, et al. Radiation dose incurred in the exclusion of vascular filling in transforaminal epidural steroid injections: fluoroscopy, digital subtraction angiography, and CT/ fluoroscopy. Pain Med 2014;15:1328-33 CrossRef Medline

11. Lazarus MS, Forman RB, Brook AL, et al. Radiation dose and procedure time for $994 \mathrm{CT}$-guided spine pain control procedures. Pain Physician 2017;20:E585-91 CrossRef Medline

12. Suresh S, Berman J, Connell A. Cerebellar and brainstem infarction as a complication of CT-guided transforaminal cervical nerve root block. Skeletal Radiol 2007;36:449-52 CrossRef Medline

13. Park GY, Kwon DR, Kwon DG. Complex regional pain syndrome type II after cervical transforaminal epidural injection: a case report. Medicine (Baltimore) 2018;97:e10784 CrossRef Medline
14. Bogduk N, Dreyfuss P, Baker R, et al. Complications of spinal diagnostic and treatment procedures. Pain Med 2008;9(Suppl 1):S11-34 CrossRef

15. Cyteval C, Thomas E, Decoux E, et al. Cervical radiculopathy: open study on percutaneous periradicular foraminal steroid infiltration performed under CT control in $\mathbf{3 0}$ patients. AJNR Am J Neuroradiol 2004;25:441-45 Medline

16. Kim MS, Lee DG, Chang MC. Outcome of transforaminal epidural steroid injection according to severity of cervical foraminal stenosis. World Neurosurg 2018;110:e398-403 CrossRef Medline

17. Costandi SJ, Azer G, Eshraghi Y, et al. Cervical transforaminal epidural steroid injections: diagnostic and therapeutic value. Reg Anesth Pain Med 2015;40:674-80 CrossRef Medline

18. Slipman CW, Lipetz JS, Jackson HB, et al. Therapeutic selective nerve root block in the nonsurgical treatment of atraumatic cervical spondylotic radicular pain: a retrospective analysis with independent clinical review. Arch Phys Med Rehabil 2000;81:741-46 CrossRef Medline

19. Vallée JN, Feydy A, Carlier RY, et al. Chronic cervical radiculopathy: lateral-approach periradicular corticosteroid injection. Radiology 2001;218:886-92 CrossRef Medline

20. Okubadejo GO, Talcott MR, Schmidt RE, et al. Perils of intravascular methylprednisolone injection into the vertebral artery: an animal study. J Bone Joint Surg Am 2008;90:1932-38 CrossRef Medline

21. Huda W, Ogden KM, Khorasani MR. Converting dose-length product to effective dose at CT. Radiology 2008;248:995-1003 CrossRef Medline

22. Lukies MW, Teoh WW, Clements W. Safety of CT-guided cervical nerve root corticosteroid injections. J Med Imaging Radiat Oncol 2019;63:300-06 CrossRef Medline

23. Depriester C, Setbon S, Larde A, et al. CT-guided transforaminal cervical and lumbar epidural injections. Diagn Interv Imaging 2012;93:70410 CrossRef Medline

24. Bartleson JD, Maus TP. Diagnostic and therapeutic spinal interventions: epidural injections. Neurol Clin Pract 2014;4:347-52 CrossRef Medline

25. Palmer W. Spinal injections for pain management. Radiology 2016;281:669-88 CrossRef Medline

26. Jeon Y, Kim S. Detection of intravascular injection during cervical transforaminal epidural injection: a comparison of digital subtraction angiography and real time fluoroscopy. Pain Physician 2018;21: E181-86 CrossRef Medline

27. Schmid G, Schmitz A, Borchardt D, et al. Effective dose of CT- and fluoroscopy-guided perineural/epidural injections of the lumbar spine: a comparative study. Cardiovasc Intervent Radiol 2006;29:8491 CrossRef Medline

28. Dietrich TJ, Peterson CK, Zeimpekis KG, et al. Fluoroscopy-guided versus CT-guided lumbar steroid injections: comparison of radiation exposure and outcomes. Radiology 2019;290:752-59 CrossRef Medline 